\title{
El impuesto sobre la renta empresarial en Colombia: su tasa efectiva y su relación con la inversión
}

\author{
Víctor Mauricio Castañeda Rodríguez* \\ Jairo Orlando Villabona**
}

Fecha de recepción: 6 de enero de 2020

Fecha de aceptación: 28 de junio de 2020

Resumen: En Colombia es tradicional que cuando se trata de emprender reformas tributarias, se indique también la necesidad de promover la inversión y el crecimiento económico, de modo que se recurre a fórmulas como la reducción de las alícuotas del impuesto sobre la renta empresarial (ISRE) y la creación de beneficios tributarios. Este trabajo revisa los postulados tras la reforma tributaria de 2018 en Colombia, conocida como Ley de Financiamiento, para mostrar, primero, que la tasa efectiva asociada al ISRE no es muy alta, para lo cual se consideran los datos agregados de las declaraciones tributarias de la Dirección de Impuestos y Aduanas Nacionales para el año 2016. En segundo lugar, y a través de un ejercicio econométrico basado en un panel de datos que incluye observaciones para 109 países (dada la insuficiencia de datos para considerar solo a Colombia) durante el periodo 2006-2015, se establece que estadísticamente no hay evidencia de una relación entre impuestos corporativos e inversión.

Palabras clave: impuesto sobre la renta empresarial; inversión; inversión extranjera directa; tasa efectiva de tributación; Ley de Financiamiento.

Clasificación JEL: H71, M21, O23, E62.

Cómo citar este artículo/ To reference this article / Comment citer cet article / Para citar este artigo:

Castañeda Rodríguez, V., \& Villabona-Robayo, J. (2020). El impuesto sobre la renta empresarial en Colombia: su tasa efectiva y su relación con la inversión. Apuntes del Cenes, 39(70). Págs. 183 - 205

https://doi.org/10.19053/01203053.v39.n70.2020.10493

\footnotetext{
* Doctor en Ciencias Económicas. Profesor asociado de la Facultad de Ciencias Económicas de la Universidad Nacional de Colombia. Bogotá, Colombia. Estudios Interdisciplinarios sobre Gestión y Contabilidad (INTERGES). Correo electrónico: vmcastanedar@unal.edu.co (iD http://orcid.org/0000-0001-8343-6739

** Doctorat en Sciences de Gestion. Profesor asociado de la Facultad de Ciencias Económicas de la Universidad Nacional de Colombia. Bogotá, Colombia. Grupo de Estudios Fiscales y de Equidad. Correo electrónico: jovillabonar@ unal.edu.co (D) http://orcid.org/0000-0002-4060-7478.
} 


\title{
The Corporate Income Tax in Colombia: its Effective Rate and its Relationship with Investment
}

\begin{abstract}
In Colombia it is traditional that discussions about tax reforms indicate that corporate taxation is so burdensome that it drives away the investment and slows down economic growth, which is why preferential tax treatments or tax cuts are promoted. This is, in fact, what is behind the most recent tax reform in Colombia, which was named as the Financing Law in 2018, but this research shows two facts. The first one is that the effective rate of Corporate Income Tax is not as high as it is stressed in Colombia by the media, economic unions and many politicians, for which we take into account tax reporting data provided by the Colombian tax administration office (DIAN) for 2016. The second issue is related to the generally accepted hypothesis that taxation discourages investment, which is denied through a data panel analysis across 109 countries (including Colombia) and the period 2006-2015.
\end{abstract}

Keywords: corporate income tax; investment; foreign direct investment; effective tax rate; financing law. 


\section{INTRODUCCIÓN}

Los impuestos son una importante fuente de recursos fiscales, además de ser un instrumento para redistribuir la riqueza y los ingresos. En consecuencia, dentro de los principios de la tributación se encuentra la progresividad y la eficiencia, pues ellos permiten establecer que normativamente sean las personas con más altos ingresos y riqueza quienes más impuestos paguen, y además suponen una premisa para perseguir el debido recaudo. No obstante, en la práctica se encuentran diferencias significativas entre sistemas tributarios, por ejemplo en los países más desarrollados la mayor fuente de recaudación por concepto de impuestos sobre la renta corresponde a las personas naturales, mientras que en Colombia (así como en la mayoría de los países de América Latina) se concentra en las empresas (Castañeda-Rodríguez, 2019).

En consonancia con lo anterior, se indica que las empresas en Colombia pagan altos impuestos, lo que trae consigo una pérdida de competitividad para el país. Por ejemplo, una revisión a la exposición de motivos de la Ley de Financiamiento de 2018 permite identificar la posición del Gobierno colombiano en torno a la tributación empresarial, pues allí se culpa a la presuntamente alta carga impositiva como uno de los factores que desincentiva la inversión y contribuye a explicar la desaceleración económica. Tampoco es difícil encontrar alocuciones de las agremiaciones empresariales del país en que se presenta al impuesto sobre la renta empresarial (ISRE) como un obstáculo para el emprendimiento y desarrollo económico.

Al respecto, este trabajo se plantea dos objetivos. Identificar si efectivamente las empresas en Colombia soportan una carga tributaria muy onerosa y verificar si la hipótesis de una relación inversa entre impuestos corporativos e inversión tiene asidero empírico. Esto se hace teniendo en cuenta que la reforma tributaria de 2018 en el país consideró que estos dos puntos eran ciertos, lo que coincide con las presunciones que tradicionalmente se hacen en relación con la tributación empresarial en Colombia y América Latina, en general. 
En cuanto al primer objetivo, en este trabajo se calcula la tasa efectiva de tributación (TET) corporativa con base en el agregado de las declaraciones de renta de las empresas para el año 2016, y se establece que esta no es alta como lo manifiestan el Gobierno, los gremios económicos y los medios de comunicación en general. Además, este resultado se confronta con otros trabajos realizados en el mismo sentido en el país, como los de Villabona y Quimbay (2017) y Gómez y Steiner (2015).

En efecto, se determina que el ISRE en Colombia no es tan alto como se asume, pues existe un conjunto de beneficios tributarios que toman diferentes formas, como en el caso de las deducciones imputables, las rentas exentas, los ingresos no grabados y los descuentos tributarios, que reducen en buena medida el monto del impuesto a cargo. Se muestra que para el año 2016 la TET del ISRE para el total de sectores económicos no superó el $24 \%$, sumando el impuesto para la equidad CREE y su sobretasa, cifras que van en la misma línea que las calculadas por Villabona y Quimbay (2017) para el periodo 2000-2015. En síntesis, estos valores son inferiores a los reportados por los gremios, el Gobierno e incluso otras publicaciones como la de Gómez y Steiner (2015), dados algunos vacíos metodológicos o supuestos tras sus estimaciones.

La segunda aseveración que se analiza en este documento se refiere a la aparente relación negativa y significativa entre impuestos corporativos e inversión, y en particular inversión extranjera directa (IED). Esta idea, que se ha convertido en casi un dogma de la tributación en Colombia desde la aplicación de las políticas de ajuste del Consenso de Washington, sugiere la necesidad de "moderar" la carga tributaria de las empresas, aun cuando ello suponga aumentar otros impuestos que pueden resultar regresivos, como el IVA; sin embargo, en este artículo se concluye que tal asociación entre impuestos e inversión no tiene asidero empírico, para lo cual se recurre a un ejercicio econométrico de tipo longitudinal que incluye observaciones para 109 países (entre ellos Colombia) durante el periodo 2006-2015, dada la insuficiencia de observaciones para considerar solo un país, i.e., Colombia.

Los resultados de dicho ejercicio contribuyen además a la discusión sobre los efectos de los impuestos en la inversión, pues indican que los inversionistas consideran en sus decisiones factores que trascienden la política fiscal de un país. Al respecto, autores como Jiménez y Podestá (2009) han indicado que variables como la estabilidad política y económica, la seguridad jurídica, el tamaño de la economía, la cualificación de la mano de obra y el grado de apertura comercial son importantes a la hora de estudiar la dinámica de la IED. Con base en ello, este artículo adiciona una amplia lista de controles para luego, tras realizar varios contrastes econométricos, inclinar la balanza a favor de 
trabajos como el de Hunady y Orviska (2014), en los que se encuentra que los impuestos corporativos no contribuyen a explicar el comportamiento de la inversión, a pesar de los hallazgos de otras investigaciones en la materia (véanse e.g., Chanegriha et al., 2017; Agostini $\&$ Jalile, 2009).

Por lo tanto, esta investigación hace dos aportes en el campo de la tributación. Por una parte, contribuye a medir la carga tributaria que las empresas efectivamente soportan en Colombia por concepto de ISRE, al seguir un proceso de depuración que tiene en cuenta los beneficios tributarios que reducen el impuesto a cargo. Además, y dada la posibilidad de diferenciar por sectores económicos en las declaraciones agregadas que provee la Dirección de Impuestos y Aduanas Nacionales (DIAN), se evidencia que este impuesto es inequitativo, tanto en la dimensión horizontal como en la vertical. Asimismo, se provee nueva evidencia empírica que pone de manifiesto que las decisiones de inversión no dependen necesariamente de factores tributarios, de modo que tienen mayor importancia otras variables como el grado de apertura comercial y la tasa de crecimiento de la economía, considerando para ello tanto modelaciones estáticas como dinámicas.

Este trabajo incluye otras cuatro secciones además de la introducción. En la primera de ellas se revisa la presunción sobre la elevada TET por concepto de ISRE en Colombia, para mostrar que no es válida a juzgar por los datos consignados en el agregado de declaraciones de renta, según información de la DIAN para el año gravable 2016. En la segunda parte se hace una breve revisión de la literatura teórica y empírica pertinente para estudiar la relación entre impuestos empresariales e inversión, la cual sirve de preámbulo para el siguiente apartado, en el que se lleva a cabo un ejercicio econométrico basado en un panel de datos con observaciones para 109 países (entre ellos Colombia) durante el periodo 2006-2015. Por último, se presentan las conclusiones del estudio.

\section{LA TASA EFECTIVA DEL ISRE EN COLOMBIA}

Los gremios empresariales en Colombia han procurado demostrar que los impuestos para las empresas son muy altos, campaña que se ha difundido ampliamente en los medios de comunicación más importantes del país. Por ejemplo, Bruce Mc Master, hasta noviembre de 2017 director del Departamento para la Prosperidad Social (entidad adscrita a la Presidencia de la República) y ahora presidente de la Asociación Nacional de Empresarios de Colombia, indicó en una entrevista con el diario El País: "Colombia tiene una tasa de tributación para los empresarios del $70 \%$, la tercera más alta del mundo, lo cual es una de sus grandes desventajas y una torpeza gigantesca [...]" (García, 2016).

Sin embargo, no hay claridad absoluta en las cifras que se suministran con 
respecto a los impuestos sobre la renta que pagan las empresas en Colombia, lo que ocasiona una gran dispersión y desinformación en las comunicaciones. Se suele afirmar en los medios que la tasa de tributación es del $70 \%$, aunque quizás se refieren a la tasa nominal sumando hasta el IVA. Además, la forma como se calcula la TET varía en cada país teniendo en cuenta que los códigos tributarios son diferentes y en consecuencia también lo es la depuración de la renta. En el caso colombiano estos cálculos pueden cambiar mucho a lo largo del tiempo, con ocasión de las frecuentes reformas que se aprueban en la materia.

Aunque algunas veces para calcular la TET se alude a agregados macroeconómicos, en esta sección se consideran los datos que presentan los contribuyentes en sus declaraciones de renta. Su cálculo corresponde al cociente entre el impuesto neto de renta y la utilidad comercial, para lo cual se debe seguir el proceso de depuración propio del ISRE. Por ejemplo, estimar la utilidad comercial con base en los agregados de las declaraciones de renta implica partir de la renta líquida gravable, sobre la cual se aplica la tarifa nominal, y sumarle las rentas exentas así como la deducción del IVA por compra de activos fijos y los ingresos que no constituyen renta.

En el numerador se toma el impuesto neto de renta y no el impuesto sobre la renta líquida gravable, pues el primero resta los descuentos tributarios que corresponden a otro beneficio fiscal. A continuación se presenta el cálculo de la TET agregada para el año 2016 con base en las precisiones ya realizadas.

$$
\begin{gathered}
\mathrm{TET}=\frac{\$ 22.146 .606}{\$ 93.077 .344+\$ 14.849 .851+\$ 2.917 .507+\$ 36.524 .136}=\frac{\$ 22.146 .606}{\$ 147.368 .837} \\
=15 \%
\end{gathered}
$$

* Cifras en millones de pesos

La TET calculada con base en los agregados de las declaraciones de renta para 2016 es del $15.0 \%$; sin embargo, es importante indicar que para ese año las empresas debían pagar el impuesto sobre la renta para la equidad CREE, cuyo propósito era sustituir los aportes que las empresas y algunas personas naturales hacían al Servicio Nacional de Aprendizaje SENA, al Instituto Colombiano del Bienestar Familiar y al sistema de salud como empleadores. La tarifa del CREE era del $9 \%$ y sobre la misma base se calculaba una sobretasa del $6 \%$, de modo que teniendo en cuenta que dichas tarifas eran nominales y aplicando el porcentaje que resulta de dividir la TET acá calculada entre la tarifa nominal del ISRE (60 \%), la primera se convierte en una tasa efectiva del $5.4 \%$ y la segunda del $3.6 \%$. Así, si se suman las tres tasas, se obtiene 
una TET total del $24 \%$. Cabría en otra ocasión comparar este dato con el que se podría extraer de las declaraciones del CREE.

Al respecto, Villabona y Quimbay (2017), con base asimismo en los agregados de las declaraciones de renta, establecieron que la TET promedio del ISRE en Colombia entre 2000 y 2015 fue del $17.3 \%$. Este valor se asimila al acá calculado, si se le suma el CREE y otras sobretasas, como ya se hizo. Además, hay otros trabajos, como el de Rincón-Castro y Delgado-Rojas (2018), que establecen que durante el período de estudio el promedio de las tasas impositivas sobre el consumo y los ingresos del trabajo y del capital fueron del $10.7 \%$, $18.6 \%$ y $15.4 \%$ respectivamente. En esa investigación se utilizaron los agregados macroeconómicos contenidos en las cuentas económicas integradas del Departamento Administrativo Nacional de Estadística, sin prestar atención al detalle por sectores económicos. No obstante, los respectivos resultados también se encuentran por debajo de lo expresado por los gremios, los políticos y los medios de comunicación en general.

Por otra parte, Gómez y Steiner (2015) estiman la TET asociada al ISRE como porcentaje de las utilidades operacionales antes de impuestos, con base en los datos suministrados por las firmas a la Superintendencia de Sociedades y no en la información de las declaraciones de renta. Los autores calculan que la TET promedio del ISRE para 2013 fue del
$29.9 \%$, valor que aún con sus inconsistencias está por debajo del informado por los gremios En efecto, entre las limitaciones de dicho estudio se puede mencionar que desconoce el total de las empresas declarantes y que asume que la provisión contable del impuesto equivale a la obligación fiscal.

Ahora bien, además de calcular la TET asociada al ISRE, también es importante contar con estimaciones por sectores económicos, en la medida que ello permite: (i) determinar y comparar la equidad horizontal del sistema tributario en los diferentes sectores de la economía; (ii) analizar qué tan alejado se encuentra el pago efectivo del impuesto de renta frente a la tasa nominal y (iii) cuantificar el nivel de ineficiencia e inequidad del sistema tributario con ocasión de los beneficios tributarios, a partir de la comparación de esta tasa con la nominal (una mayor diferencia implica una mayor ineficiencia e inequidad).

En consecuencia, se repite el procedimiento señalado al comienzo de esta sección, pero filtrando los agregados de las declaraciones de renta del año gravable 2016 por sector económico. Con base en esto, se puede observar que los sectores más importantes para la generación de empleo del país, como el sector industrial y el agrícola, contribuyen con TET más altas, $20.1 \%$ y $18.7 \%$ respectivamente. Por otro lado, el sector financiero reporta una TET del $11.4 \%$ (en ambos casos sin incluir el CREE). La conclusión que se desprende 
de esto es que los beneficios tributarios en Colombia no incentivan a los sectores que pudieran impulsar el empleo y crecimiento económico, sino que favorecen a aquellos que no tienen una lógica productiva, lo que debería servir como fundamento para replantear las políticas tributarias en el país.

Tabla 1. Tasas efectivas de tributación sectoriales para el impuesto sobre la renta empresarial, año 2016 (cifras en billones de pesos)

\begin{tabular}{|c|c|c|c|c|c|c|c|c|}
\hline Actividad & $\begin{array}{l}\text { Ingresos } \\
\text { no renta }\end{array}$ & $\begin{array}{l}\text { Deducción } \\
\text { inversión } \\
\text { activos }\end{array}$ & $\begin{array}{l}\text { Rentas } \\
\text { exentas }\end{array}$ & $\begin{array}{c}\text { Renta } \\
\text { líquida } \\
\text { gravable } \\
\text { (RLG) }\end{array}$ & $\begin{array}{l}\text { Impuesto } \\
\text { sobre la } \\
\text { RLG }\end{array}$ & Descuentos & $\begin{array}{l}\text { Impuesto } \\
\text { neto de } \\
\text { renta }\end{array}$ & TET \\
\hline $\begin{array}{l}\text { Agricultura y } \\
\text { ganadería }\end{array}$ & 0.4 & 0.0 & 0.1 & 1.6 & 0.4 & 0.00 & 0.4 & $18.7 \%$ \\
\hline Minería & 5.3 & 0.0 & 0.0 & 7.8 & 1.9 & 0.04 & 1.9 & $14.4 \%$ \\
\hline Manufactura & 1.7 & 0.2 & 0.6 & 17.4 & 4.1 & 0.12 & 4.0 & $20.1 \%$ \\
\hline $\begin{array}{l}\text { Electricidad, gas, } \\
\text { vapor y agua }\end{array}$ & 1.6 & 0.8 & 0.5 & 7.9 & 2.0 & 0.16 & 1.8 & $16.7 \%$ \\
\hline Construcción & 0.6 & 0.4 & 1.0 & 5.3 & 1.3 & 0.01 & 1.3 & $17.8 \%$ \\
\hline $\begin{array}{l}\text { Comercio y } \\
\text { servicios }\end{array}$ & 7.3 & 0.5 & 0.7 & 24.7 & 6.2 & 0.06 & 6.0 & $18.0 \%$ \\
\hline $\begin{array}{l}\text { Información y } \\
\text { comunicaciones }\end{array}$ & 0.4 & 0.7 & 0.3 & 3.9 & 0.9 & 0.03 & 0.9 & $17.6 \%$ \\
\hline Financieras & 11.1 & 0.1 & 4.7 & 13.8 & 3.4 & 0.04 & 3.4 & $11.4 \%$ \\
\hline $\begin{array}{l}\text { Actividades } \\
\text { profesionales, } \\
\text { científicas }\end{array}$ & 2.9 & 0.1 & 0.2 & 6.6 & 1.5 & 0.03 & 1.5 & $15.3 \%$ \\
\hline
\end{tabular}

Fuente: elaboración propia con base en agregados de las declaraciones de renta, personas jurídicas, año gravable 2016 DIAN. Los cálculos no incluyen el CREE ni la sobretasa.

Una vez se ha establecido que el ISRE no es tan alto en Colombia como normalmente se asume, se debe revisar si hay evidencia de que los impuestos corporativos desincentivan la inversión, pues de ser así, habría una justificación para que en las últimas reformas tributarias se haya promovido su disminución. En consecuencia, la siguiente sección hace una revisión general de la literatura teórica y empírica pertinente para estudiar la relación entre impuestos empresariales e inversión, la cual sirve de preámbulo para la constatación empírica que aparece más adelante.

\section{REVISIÓN DE LITERATURA ACERCA DE LOS EFECTOS DE LA TRIBUTACIÓN SOBRE LA INVERSIÓN}

Los impuestos, al alterar los precios de los factores y los posibles retornos de la inversión y el trabajo, según el caso, pueden asimismo incidir en las decisiones económicas de los hogares, las empresas y el Gobierno. Al respecto, los desarrollos teóricos en la materia han sido prolíficos, especialmente entre los sesenta y setenta, gracias al interés académico por definir sistemas tributarios 
óptimos que maximizaran el bienestar social. Por ejemplo, Alm (1996) hace una buena síntesis de las principales conclusiones de dicha literatura, aunque también llama la atención sobre las complejidades que supone la aplicación de un código tributario y la gestión de la recaudación, por lo que no siempre los resultados de la literatura normativa se pueden tomar como una guía práctica.

En efecto, Atkinson y Stiglitz (2015) en los resultados que presentan a través de varios capítulos sobre los efectos económicos y financieros de la tributación (e.g., en cuanto a la inversión) hacen también énfasis en las condiciones particulares en que un determinado resultado se podría dar. Esto, en otros términos, indica lo difícil que es encontrar hechos estilizados que desde la teoría sean válidos bajo diferentes escenarios fiscales, por ejemplo, métodos alternativos de depreciación, la aceptación fiscal de la deducción por pago de intereses, la incorporación de créditos fiscales o el grado de integración entre el ISRE y el ISRP, solo para citar algunos casos.

Es un resultado microeconómico bien conocido que la demanda de capital y trabajo depende de la productividad marginal de cada uno de estos factores, de modo que la inversión experimenta cambios de un periodo a otro dependiendo del efecto que tengan, entre otros, los impuestos corporativos sobre el costo efectivo del capital; sin embargo, el costo del capital no es afectado por los impuestos, si los incrementos marginales de la inversión se financian a través de deuda y se provisiona el total de la depreciación económica (Atkinson \& Stiglitz, 2015), lo cual corresponde a un escenario teórico en que los impuestos corporativos no inciden en la inversión. Aunque este hallazgo también se podría obtener cuando no se introducen condiciones sobre el monto de la depreciación y los intereses no son deducibles, es claro que hay otros escenarios en que la aparente neutralidad de la política tributaria en torno a la inversión desaparece. Esto corrobora la idea de que la literatura teórica no es concluyente en cuanto a la relación ISRE-inversión, pues ella está sujeta a la modelación que se considere.

También cabe precisar que la multitud de factores en juego hace difícil identificar relaciones claras entre inversión e impuestos empresariales. Al respecto, la existencia de costos de ajuste y costos hundidos dificulta modificar la cantidad de factores productivos en función de los cambios impositivos, de modo que es poco probable que la inversión responda, al menos a corto plazo, a pequeños ajustes en la carga tributaria o al diferencial de impuestos entre países. A ello habría que sumar la distribución de potestades tributarias entre los países de fuente y de residencia (i.e., modelos de tributación internacional) y el grado de incertidumbre sobre los cambios en la normatividad fiscal. 
A pesar de lo anterior, algunos modelos de política económica como el llamado "Consenso de Washington", en que la inversión es uno de los principales motores del crecimiento económico, insisten en que los impuestos corporativos constituyen un obstáculo para la misma. Las reformas económicas que se promueven desde la ortodoxia económica generalmente son favorables a la inversión (e.g., liberalización comercial, de las tasas de interés y de la IED, junto a la promoción de tasas de cambio competitivas) y para ello, desde el ámbito tributario, se recomienda la aplicación de esquemas con amplias bases y tasas marginales moderadas, pues se asume que los altos impuestos directos representan un desincentivo para los inversionistas y las empresas. En consecuencia, y como base de las propuestas de reforma tributaria, se termina entonces sugiriendo la reducción de las alícuotas marginales máximas del ISRE y la compensación de la pérdida de recaudo a través del aumento del IVA (Castañeda-Rodríguez, 2016).

Al respecto, cabe recordar que el proyecto inicial de la Ley de Financiamiento soportaba buena parte de la meta de mayor recaudación en una generalización del IVA, receta seguida comúnmente en varios países de América Latina cuando se trata de controlar el déficit fiscal en situaciones de desaceleración económica (Castañeda-Rodríguez, 2016). Esta iniciativa incluía un esquema de devolución del impuesto pagado por los deciles más pobres de la sociedad, mientras que en el caso del ISRE se proponía una reducción generalizada; sin embargo, el trámite legislativo del proyecto de ley condujo a que algunas medidas fueran excluidas, como la generalización del IVA, del mismo modo como ocurrió en 2006 con una reforma tributaria similar.

Ahora conviene revisar los hallazgos de la literatura empírica, dadas las limitaciones de los modelos teóricos para anticipar con claridad los efectos de los impuestos empresariales sobre la inversión; sin embargo, en este frente también faltan resultados concluyentes. Por una parte, se indica que en el mejor de los casos los impuestos podrían jugar un rol secundario sobre la inversión, pues los inversionistas tienden a considerar muchos otros elementos en sus decisiones (Hunady \& Orviska, 2014; Jiménez \& Podestá, 2009). Por ejemplo, otros factores que la literatura ha considerado relevantes para explicar la dinámica de la inversión son la estabilidad política y económica, la seguridad jurídica, la cualificación de la mano de obra, el tamaño de la economía y el grado de apertura comercial.

Asimismo, hay estudios que desde lo cualitativo, como el del Banco Mundial (2018), han identificado que los agentes basan sus decisiones de inversión principalmente en factores como la estabilidad política e institucional del país, de modo que los impuestos y bajos costos laborales pasan a un segundo lugar. 
Dicho trabajo se basó en una encuesta practicada a 750 ejecutivos e inversionistas multinacionales.

Por el contrario, otros estudios indican que los altos impuestos corporativos desincentivan la inversión y en particular la IED (Chanegriha et al., 2017; Agostini \& Jalile, 2009). Es bajo esta idea que se erigen los estudios sobre competencia tributaria, en que se considera que los países pueden utilizar la política fiscal para atraer la inversión extranjera a través de la incorporación de tratamientos diferenciales que reduzcan la tasa efectiva de tributación (e.g., reducciones de las tasas estatutarias acompañadas de la creación de rentas exentas o esquemas de diferimiento de las obligaciones tributarias). Pero, de nuevo, una revisión de la literatura empírica muestra que hay tanto evidencia a favor como en contra para la relación entre el grado de integración económica y las tarifas aplicables a las rentas corporativas, discusión que se desarrolla en profundidad en Adam et al. (2013).

\section{EJERCICIO EMPÍRICO: LA CAR- GA TRIBUTARIA EMPRESARIAL Y LA INVERSIÓN}

Parte de las reformas tributarias que se presentan en un país en un contexto de desaceleración económica aducen a la necesidad de promover la inversión, para lo cual una estrategia usualmente empleada consiste en reducir la carga tributaria que soportan las empresas.
En el caso concreto de Colombia, la exposición de motivos de la Ley de Financiamiento indica que hay dos factores que explican la reducción del potencial de crecimiento de la economía, se trata de la caída en la inversión y el bajo crecimiento en la productividad total de los factores. Con relación a la inversión, dicho documento explica que esta "[...] enfrenta varios obstáculos y altos costos que la desincentivan [...]. Uno de ellos es la alta carga tributaria empresarial, ya que según el Doing Business 2018 la tasa efectiva de tributación se encuentra en $69,7 \%$ de las utilidades brutas [...]" (Ministerio de Hacienda y Crédito Público, 2018, p. 37).

Este apartado pretende analizar desde un enfoque econométrico la relación entre impuestos corporativos e inversión, a pesar de lo ya comentado en la sección anterior. Ya se ha mostrado que la TET del ISRE en Colombia es inferior a la que presentan actores como el Gobierno y los gremios económicos, pero ahora es necesario validar si eventualmente un aumento del impuesto podría dar lugar a una caída de la inversión en general o de la IED en particular.

\section{Factores relacionados con la inversión}

Se debe tener en cuenta que estudiar la relación entre inversión, ya sea total o solo extranjera, y la carga tributaria empresarial resulta difícil, pues los datos disponibles en general hacen referencia a agregados que no consideran la multi- 
tud de tratamientos que un sistema tributario puede dar a un determinado sector o tipo de contribuyente. Si bien hay un conjunto de minoraciones impositivas que son generales, otras dependen del sector o tipo de contribuyente, lo cual no es capturado por las tasas de tributación promedio. Por ejemplo, en Colombia las rentas provenientes de la prestación de servicios de hotelería son gravadas con una tarifa reducida del $9 \%$ en relación con aquellos ingresos generados por los inmuebles construidos o ampliados entre 2003 y 2017 (en comparación con la tarifa general de $32 \%$ en 2020).

A pesar de lo anterior, esta sección analiza la relación entre impuestos e inversión a partir de diferentes especificaciones econométricas y variables. La Tabla 2 presenta el conjunto de variables que se emplearán en el ejercicio econométrico que sigue, de acuerdo con la revisión bibliográfica realizada, además de la descripción de las mismas, los signos esperados de las respectivas relaciones y las bases de datos a las que se acudió. Si no se puede anticipar el signo de la asociación entre variables por la falta de consenso en la literatura, como ocurre con los impuestos y la inversión, se incluye un signo de interrogación.

Las primeras dos variables explicativas de la Tabla 2 constituyen alternativas para capturar la carga tributaria empresarial. Se trata del total de impuestos y contribuciones obligatorias que en promedio una empresa debe pagar du- rante su segundo año de actividades, así como la carga que debe soportar solo por concepto del ISRE, en ambos casos como porcentajes de su utilidad comercial. Se toman las mediciones provistas por el Banco Mundial, a través de Doing Business (2019), por dos razones: su disponibilidad para un amplio número de países y por servir de referencia en diversos estudios.

La Tabla 2 además clasifica los posibles factores asociados con la inversión en grupos, para así resumir los principales argumentos que la literatura expone sobre su relevancia. Un inversionista que decide ingresar a un mercado tendrá en cuenta aspectos institucionales como la forma en que opera el Gobierno, el contexto político del respectivo país o el grado de intervención estatal, pues ellos incidirán en la probabilidad de obtener utilidades, las garantías de no ser expropiado y el grado de decisión en asuntos como contratación y despido de personal, entre otros aspectos (véanse e.g., Hunady \& Orviska, 2014; Jiménez \& Podestá, 2009). Así, por ejemplo, una Administración pública poco efectiva supone un riesgo de dilación de los procesos que requieren del aval o aprobación de alguna autoridad estatal, como en el caso de la expedición de una licencia de operación, o incluso la posibilidad de que un funcionario público decida con base en criterios no solo técnicos, lo cual representa un contexto poco favorable para la inversión. 
Tabla 2. Variables objeto de análisis econométrico

\begin{tabular}{|c|c|c|c|}
\hline & Variable & Descripción & Base de datos \\
\hline \multirow{2}{*}{ 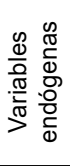 } & IED & $\begin{array}{l}\text { Entradas netas de inversión a cargo de no residentes dedicadas a } \\
\text { adquirir el control de al menos el } 10 \% \text { de las acciones o derechos } \\
\text { a voto de una empresa domiciliada en el país (porcentaje del PIB). }\end{array}$ & $\begin{array}{l}\text { Banco Mundial } \\
\text { (Indicadores de } \\
\text { desarrollo) }\end{array}$ \\
\hline & Inversión_total & Formación bruta de capital como porcentaje del PIB. & $\begin{array}{l}\text { Fondo Monetario } \\
\text { Internacional }\end{array}$ \\
\hline \multirow{2}{*}{$\begin{array}{l}\frac{0}{0} \\
\frac{0}{0} \\
\frac{0}{\underline{E}}\end{array}$} & $\begin{array}{c}\text { Carga_tributaria_total } \\
(?)\end{array}$ & $\begin{array}{l}\text { Total de impuestos que una firma debe pagar (porcentaje de su } \\
\text { utilidad bruta). }\end{array}$ & \multirow[t]{2}{*}{$\begin{array}{l}\text { Banco Mundial } \\
\text { (Doing Business) }\end{array}$} \\
\hline & Carga_ISRE (?) & $\begin{array}{l}\text { Impuestos sobre la renta empresarial (porcentaje de su utilidad } \\
\text { bruta). }\end{array}$ & \\
\hline \multirow{4}{*}{ 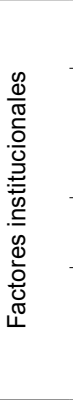 } & $\begin{array}{c}\text { Estabilidad_política* } \\
(+)\end{array}$ & Índice de estabilidad política y ausencia de violencia. & \multirow{3}{*}{$\begin{array}{l}\text { Banco Mundial } \\
\text { (Indicadores de } \\
\text { gobernanza) }\end{array}$} \\
\hline & $\begin{array}{l}\text { Efectividad } \\
\text { gobierno* }(+)\end{array}$ & $\begin{array}{l}\text { Índice que captura la percepción de expertos sobre la calidad de } \\
\text { la burocracia y de los servicios que provee el Estado, además } \\
\text { del grado de credibilidad e independencia con que actúa la } \\
\text { Administración pública. }\end{array}$ & \\
\hline & $\underset{(+)}{\text { Control_corrupción* }}$ & $\begin{array}{l}\text { Índice de percepción sobre cuán instituida está la corrupción en el } \\
\text { ejercicio del poder público. }\end{array}$ & \\
\hline & Regulación** (+) & $\begin{array}{l}\text { Índice del grado de regulación estatal sobre el sistema bancario, el } \\
\text { mercado laboral, las tasas de interés y el sistema de precios en la } \\
\text { economía. El rango de valores está entre } 0 \text { y } 10 .\end{array}$ & $\begin{array}{l}\text { Fraser Institute } \\
\text { (Base de Datos } \\
\text { sobre Libertad } \\
\text { Económica) }\end{array}$ \\
\hline \multirow{4}{*}{ 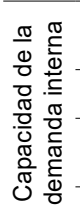 } & PIB_per_cápita (+) & $\begin{array}{l}\text { Producto interno bruto per cápita medido en dólares con valor } \\
\text { adquisitivo constante de } 2010 \text {. }\end{array}$ & \multirow{8}{*}{$\begin{array}{l}\text { Banco Mundial } \\
\text { (Indicadores de } \\
\text { Desarrollo) }\end{array}$} \\
\hline & Crecimiento $(+)$ & Tasa de crecimiento del PIB entre dos años consecutivos. & \\
\hline & Desempleo (-) & $\begin{array}{l}\text { Tasa de desempleo (porcentaje de la fuerza laboral) con base en } \\
\text { estimaciones de la Organización Internacional del Trabajo. }\end{array}$ & \\
\hline & Población (+) & Número de habitantes medido en miles de personas. & \\
\hline & Apertura (+) & Suma de importaciones y exportaciones (porcentaje del PIB). & \\
\hline 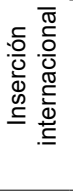 & Rentas_naturales & $\begin{array}{l}\text { Total de rentas obtenidas por la explotación de petróleo, gas natural, } \\
\text { carbón, minerales en general y recursos forestales (porcentaje del } \\
\text { PIB). Dichas rentas corresponden al producto de la cantidad de } \\
\text { unidades extraídas (o cosechadas) por la diferencia entre el precio } \\
\text { por unidad del respectivo bien (e.g., barril de petróleo) y su costo } \\
\text { promedio de producción. }\end{array}$ & \\
\hline \multirow{2}{*}{ 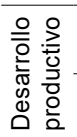 } & Educación (+) & $\begin{array}{l}\text { Tasa de matrícula bruta en educación terciaria (técnica y } \\
\text { profesional). }\end{array}$ & \\
\hline & Agricultura (-) & $\begin{array}{l}\text { Participación de la agricultura, incluyendo ganadería, silvicultura, } \\
\text { caza y pesca, en el PIB. }\end{array}$ & \\
\hline
\end{tabular}

Fuente: elaboración propia con base en la revisión, por ejemplo, de Agostini y Jalile (2009), Jiménez y Podestá (2009), Hunady y Orviska (2014) y Chanegriha et al. (2017).

Notas: *Los indicadores de gobernanza se distribuyen normalmente con media cero y desviación estándar de uno, por lo que sus valores pertenecen al rango entre -2.5 y 2.5 ; los valores más altos se asocian con mejores resultados (e.g., mayor estabilidad política). ${ }^{* *}$ Un valor de 10 para la variable Regulación es consistente con un escenario en que existe un alto porcentaje de depósitos en bancos de propiedad privada, la tasa de negación de licencias solicitadas por bancos extranjeros es baja, la participación del sector privado en la oferta de crédito se acerca al máximo del año base, las tasas de interés se determinan por las fuerzas del mercado y son positivas en términos reales, las normas laborales son laxas en relación con la fijación de salarios mínimos, no hay controles estatales a los precios y, en general, comenzar un nuevo negocio es fácil. 
Asimismo, las oportunidades que presenta una economía para un inversionista, residente o foráneo, están en parte relacionadas con las expectativas de crecimiento económico, el tamaño de su mercado y la capacidad de compra de sus habitantes, variables que se agrupan bajo el título "Capacidad de la demanda interna" (Chanegriha et al., 2017); sin embargo, la mayor producción generada por las inversiones reales no siempre se dirigirá al mercado local, de suerte que el grado de inserción internacional de un país, capturado a través del índice de apertura económica, también sirve de referencia de cuán fácil es exportar bienes y servicios o acceder a insumos que no se producen localmente.

Se incluyen también las rentas por la explotación de recursos naturales en la agrupación de inserción internacional, pues las exportaciones de un país y su situación económica dependen en ciertos casos del mercado internacional de commodities. Además, esta variable sirve de proxy de la rentabilidad de la extracción de materias primas, que suele ser un renglón especialmente llamativo para la inversión en América Latina. Ella tiende a dirigirse hacia los países ricos en recursos naturales, cuando el contexto internacional garantiza altos retornos debido al incremento de los respectivos precios (Bokpin et al., 2015).

El grado de progreso tecnológico de un país o las características de su sistema de producción son también aspectos importantes para un inversionista, toda vez que de ellos dependen la calidad y el costo de los bienes y servicios que se produzcan, lo cual explica su incorporación en la literatura (véase e.g., Villaverde \& Maza, 2015). Acá se recurre a dos variables para capturar el nivel de desarrollo productivo de un país, se trata de la tasa de matrícula para la educación terciaria y la participación del sector agrícola en el PIB. En relación con el último factor, se parte de la idea de que la producción agrícola en general no requiere de insumos especializados ni es un impulsor tan eficaz de la innovación como lo son otras actividades (e.g., la fabricación industrial), de modo que el signo de su coeficiente se espera que sea negativo.

Por su parte, la Tabla 3 presenta algunas estadísticas básicas de las variables incluidas en la Tabla 2. Es de resaltar que acá se opta por un panel de datos que incluye un total de 109 países, entre ellos Colombia ${ }^{1}$, y cubre el periodo

1 Dichos países son: Albania, Alemania, Arabia Saudita, Argelia, Armenia, Austria, Azerbaiyán, Bahréin, Bangladesh, Bélgica, Belice, Benín, Bután, Botsuana, Brasil, Brunei, Bulgaria, Burkina Faso, Burundi, Camboya, Camerún, Cabo Verde, Chad, Chile, China, Colombia, Costa Rica, Costa de Marfil, Croacia, Chipre, República Checa, Ecuador, Egipto, El Salvador, Eslovaquia, Emiratos Árabes Unidos, Estonia, Etiopía, Filipinas, Francia, Georgia, Ghana, Guatemala, Guinea, Guinea-Bissau, Guyana, Honduras, Hungría, India, Indonesia, Irán, Irlanda, Israel, Italia, Jamaica, Japón, Jordania, Kazajstán, Kenia, Kuwait, Kirguistán, Laos, Letonia, Líbano, Lituania, Madagascar, Malawi, Malasia, Malí, Marruecos, Mauritania, Mauricio, México, Moldavia, Montenegro, Mozambique, Nepal, Níger, Omán, Países Bajos, Pakistán, Panamá, Paraguay, Perú, Polonia, Portugal, Catar, República Dominicana, Reino Unido, Ruanda, Rumania, Senegal, Serbia, Sri Lanka, Suecia, Suiza, Tailandia, Tanzania, Tayikistán, Timor-Leste, Togo, Túnez, Turquía, Uganda, Uruguay, Vietnam, Yemen, Zambia, Zimbabue. 
2006-2015. Se eligió este enfoque para incrementar el número de observaciones y brindar un mayor grado de confianza en las estimaciones econométricas que se presentan más adelante. $\mathrm{Si}$ se hubiera considerado solo el caso de
Colombia, se contaría con a lo sumo 30 observaciones, teniendo en cuenta que la medición de algunos factores institucionales es relativamente reciente y data de los noventa.

Tabla 3. Estadísticas básicas de las variables empleadas en el ejercicio empírico

\begin{tabular}{|c|c|c|c|c|c|}
\hline Variable & Media & $\begin{array}{l}\text { Desviación } \\
\text { estándar }\end{array}$ & Mínimo & Máximo & Observaciones \\
\hline IED & 4.657 & 4.362 & -15.989 & 26.526 & 770 \\
\hline Inversión_total & 25.556 & 8.480 & 6.348 & 73.002 & \\
\hline Carga_tributaria_total & 42.506 & 16.932 & 8 & 98.7 & \\
\hline Carga_ISRE & 15.405 & 8.508 & 0 & 34.9 & \\
\hline Estabilidad_política & -0.198 & 0.849 & -2.483 & 1.418 & 770 \\
\hline Efectividad_gobierno & -0.026 & 0.788 & -1.535 & 2.112 & \\
\hline Control_corrupción & -0.141 & 0.834 & -1.486 & 2.292 & \\
\hline Regulación & 6.980 & 0.916 & 3.611 & 8.956 & 653 \\
\hline PIB_per_cápita & 10479.61 & 15409.40 & 235.705 & 76682.64 & 768 \\
\hline Crecimiento & 4.027 & 3.445 & -7.652 & 15.446 & 770 \\
\hline Desempleo & 7.385 & 4.552 & 0.14 & 21.909 & \\
\hline Población & 33138.33 & 120482.80 & 290.75 & 1378665 & \\
\hline Apertura & 86.035 & 33.453 & 19.459 & 174.159 & \\
\hline Rentas_naturales & 8.153 & 10.605 & 0.001 & 56.982 & \\
\hline Educación & 31.614 & 20.436 & 0.476 & 67.866 & \\
\hline Agricultura & 12.042 & 10.466 & 0.094 & 46.549 & \\
\hline
\end{tabular}

Fuente: elaboración propia. Las estadísticas se obtuvieron tras depurar la base de datos; por ejemplo, al considerar solo los casos en que se tuvieran observaciones disponibles para todas las variables.

\section{Estimaciones econométricas}

A efectos del ejercicio que ahora nos ocupa, es importante indicar que existen diversas especificaciones para paneles de datos estáticos ${ }^{2}$, pues se debe controlar por el carácter individual de cada país, dada la posible existencia de factores no observables, así como evaluar si las diferencias entre secciones cruzadas son aleatorias con respecto a una media o si, por el contrario, son constantes. En otras palabras, la idea es partir de un modelo de datos agrupados, donde cada observación se toma como independiente, para posteriormente considerar si los modelos de efectos aleatorios y fijos son preferibles y finalmente seleccionar solo uno de ellos. Las estructuras generales de tales modelos se presentan en las ecuaciones [1] (Datos Agrupados-DA), [2] (Efectos Aleatorios-EA) y [3]

2 Se considera este tipo de panel de datos como una primera aproximación al análisis sobre los factores determinantes de la inversión. 
(Efectos Fijos-EF), donde i representa a cada unidad transversal de análisis (i.e., país), t corresponde al año de observación, $x_{k}$ es cada una de las $\mathrm{k}$ variables exógenas, y representa a la inversión, $\alpha$ es una constante, $u_{i}$ es una desviación aleatoria y $v_{i}$ corresponde a un coeficiente único para cada país i.

$$
\begin{gathered}
y_{i t}=\alpha+\sum_{k=1}^{K} \beta_{k} x_{k i t}+e_{i t} \\
y_{i t}=\alpha_{i}+\sum_{k=1}^{K} \beta_{k} x_{k i t}+e_{i t} \\
\operatorname{con} \alpha_{i}=\alpha+u_{i} \\
y_{i t}=v_{i}+\sum_{k=1}^{K} \beta_{k} x_{k i t}+e_{i t}
\end{gathered}
$$

El Anexo 1 presenta ocho modelos, cuatro de efectos fijos y otros cuatro de efectos aleatorios, en que se intercambian las medidas de inversión consideradas en este estudio (IED e Inversión_total) y las dos alternativas de medición para la carga tributaria empresarial ya mencionadas (Tabla 2). Aunque dichas estimaciones se deben tomar solo como intermedias, pues no corrigen algunos problemas como el de heterocedasticidad y correlación, tanto serial como contemporánea, si permiten identificar un par de asociaciones que se mantienen significativas en general. Se trata de los casos inversión-crecimiento e inversión-apertura comercial, ambas positivas por lo demás.

La Tabla 4 presenta las estadísticas obtenidas para la Prueba del Multiplicador de Lagrange, la Prueba F, los tests de Hausman y de Wooldridge, la Prueba modificada de Wald y la Prueba de Bresch y Pagan, para cada uno de los cuatro posibles modelos que subyacen al Anexo 1 (según la variable endógena y de impuestos que se incluya en cada caso). Las primeras tres pruebas se emplean para elegir entre alguna de las especificaciones representadas por las ecuaciones [1], [2] y [3], mientras que las otras tres permiten indagar sobre el cumplimiento de algunas características que deben exhibir los términos de error, ello para garantizar que los coeficientes estimados en los modelos lineales sean confiables (i.e., homocedasticidad $\mathrm{e}$ independencia serial y contemporánea).

Tabla 4. Valores p de las pruebas de selección y diagnóstico de los modelos estáticos

\begin{tabular}{ccccccc}
\hline \multicolumn{1}{c}{ Modelo } & \multicolumn{7}{c}{ Prueba } \\
\cline { 2 - 7 } & $\begin{array}{c}\text { Multiplicador } \\
\text { de Lagrange }\end{array}$ & Prueba F & Hausman & Wooldridge & $\begin{array}{c}\text { Bresch y } \\
\text { Pagan }\end{array}$ & Wald \\
\hline IED- Carga_tributaria_total & 0.000 & 0.000 & 0.000 & 0.000 & 0.000 & 0.000 \\
\hline IED-Carga_ISRE & 0.000 & 0.000 & 0.000 & 0.000 & 0.000 & 0.000 \\
\hline $\begin{array}{c}\text { Inversión_total- } \\
\text { Carga_tributaria_total }\end{array}$ & 0.000 & 0.000 & 0.000 & 0.000 & 0.000 & 0.000 \\
\hline Inversión_total-Carga_ISRE & 0.000 & 0.000 & 0.000 & 0.000 & 0.000 & 0.000 \\
\hline
\end{tabular}

Fuente: elaboración propia con base en pruebas realizadas en Stata 15. 
En la Tabla 4 se establece que independientemente de cuál sea la variable endógena y de impuestos empleada, la Ecuación 3 (i.e., Efectos Fijos) es la que mejor se ajusta a las particularidades de los datos. Además, se identifican algunos problemas de heterocedasticidad y correlación, tanto serial como contemporánea. Por lo tanto, se recurre a los errores estándar corregidos para panel (PCSE, por su acrónimo en inglés) como técnica final de estimación estática, en que se incluye también una estructura autorregresiva para tener en cuenta la correlación serial ya diagnosticada. Aunque existen otras técnicas, como la de mínimos cuadrados generalizados factibles, que se pueden emplear para tratar con la heterocedasticidad y correlación en paneles de datos, se prefirió usar PCSE dado que ello permite obtener estimaciones más confiables, especialmente cuando se dispone de muestras pequeñas (Beck \& Katz, 1995).

Por otra parte, la Tabla 5 muestra los resultados de las estimaciones PCSE, según las variables de inversión e impuestos por las que se opte. Es importante indicar que dada la diferencia de escala de algunas variables y su alta variabilidad, fue necesario también calcular los logaritmos de ellas, de suerte que los coeficientes asociados al PIB per cápita y al tamaño de la población se pueden interpretar como semielasticidades.

Tabla 5. Factores asociados con la IED y la inversión total (estimaciones PCSE)

\begin{tabular}{|c|c|c|c|c|}
\hline \multirow{2}{*}{$\begin{array}{c}\text { Variable endógena } \\
\text { Variable / Modelo }\end{array}$} & \multicolumn{2}{|c|}{ IED } & \multicolumn{2}{|c|}{ Inversión_total } \\
\hline & $1^{*}$ & $2^{*}$ & $3^{*}$ & $4^{*}$ \\
\hline Carga_tributaria_total & 0.012 & & -0.001 & \\
\hline Carga_impuestos_renta & & 0.035 & & 0.134 \\
\hline Estabilidad_política & $-1.010^{* *}$ & $-0.850^{*}$ & $1.182^{*}$ & $1.054^{*}$ \\
\hline Efectividad_gobierno & 0.661 & 0.687 & -1.322 & -1.057 \\
\hline Control_corrupción & 0.945 & 0.570 & $2.222^{*}$ & $2.074^{*}$ \\
\hline Regulación & $0.882^{* *}$ & $0.789^{*}$ & 0.658 & 0.767 \\
\hline LN(PIB_per_cápita) & $4.414^{*}$ & $4.555^{*}$ & $10.347^{* * *}$ & $10.833^{* * *}$ \\
\hline Crecimiento & $0.158^{* * *}$ & $0.161^{* * *}$ & $0.225^{\star * *}$ & $0.241^{* * *}$ \\
\hline Desempleo & -0.096 & -0.088 & $-0.498^{* * *}$ & $-0.504^{* * *}$ \\
\hline LN(Población) & $10.707^{* * *}$ & $11.929^{* * *}$ & $22.059^{* * *}$ & $21.815^{* * *}$ \\
\hline Apertura & $0.037^{\star *}$ & $0.040^{* *}$ & $0.142^{* \star *}$ & $0.140^{* * *}$ \\
\hline Rentas_naturales & 0.035 & 0.000 & $-0.224^{* * *}$ & $-0.208^{* * *}$ \\
\hline Educación & $0.092^{* *}$ & $0.087^{* *}$ & $0.080^{* *}$ & $0.088^{* * *}$ \\
\hline Agricultura & $-0.219^{* *}$ & $-0.206^{* *}$ & $-0.262^{* *}$ & $-0.268^{* * *}$ \\
\hline $\mathrm{N}$ & 622 & 634 & 556 & 568 \\
\hline r2 & 0.828 & 0.826 & 0.986 & 0.986 \\
\hline
\end{tabular}

Fuente: elaboración propia. *Significativo al $10 \%$, * Significativo al $5 \%$, ***Significativo al $1 \%$.

Nota: Las estimaciones incluyeron numerosos efectos fijos para las secciones cruzadas (países) y años, de modo que los respectivos coeficientes no se incorporaron en la tabla. 


\section{ANÁLISIS DE LOS RESULTADOS}

Lo primero que llama la atención de estos resultados es que independiente de la especificación, no parece existir una relación estadísticamente significativa entre impuestos corporativos e inversión. Por otra parte, las asociaciones entre inversión, por un lado, y tanto crecimiento económico como apertura comercial, por otro, se mantienen positivas y relevantes, como ya lo anticipaban las estimaciones preliminares (Anexo 1). La Tabla 5 también indica que a medida que la regulación estatal se torna amigable con el mercado, tiende asimismo a crecer la IED (modelos $1 *$ y $2 *$ de la Tabla 5), aunque dicha asociación pierde significancia estadística cuando en las regresiones se reemplaza a la IED por la inversión total.

La Tabla 5 además muestra que los factores relacionados con el grado de desarrollo productivo de una economía, a saber, matrícula en educación terciaria y participación de la agricultura en el PIB, se relacionan con la inversión de acuerdo con lo esperado. En otras palabras, esto sugiere que las decisiones de inversión se asocian con la dotación de factores productivos que presenta un país, así como con su capacidad de demanda (a juzgar por los coeficientes que acompañan a los logaritmos del PIB per cápita y del tamaño poblacional), lo que coincide con lo propuesto por autores como Jiménez y Podestá (2009). En general, nuestros resultados cumplen las expectativas construidas con base en la revisión de la literatura y sintetizadas en la Tabla 2.

Nuestras estimaciones indican (en particular el Modelo 1* de la Tabla 5), entre otras cosas, que un aumento del $1 \%$ en el PIB per cápita se asocia con un incremento del $0.044 \%$ de la IED. En general, los resultados de la Tabla 5 tomados en su conjunto sugieren que la inversión se beneficia de factores que dan cuenta de la buena situación económica de un país, la claridad y certidumbre que brindan sus instituciones en actividades de regulación, su grado de apertura comercial y el grado de desarrollo de su estructura productiva ${ }^{3}$.

Sin embargo, es posible que un lector se pregunte si estos resultados son confiables, pues no se han considerado los problemas de endogeneidad que podrían existir, así como la eventual dependencia de la trayectoria de la inversión, extranjera o total, lo que invita a considerar también modelos dinámicos. Para esto, se pueden reestimar los modelos de la Tabla 5, pero empleando los estimadores de Arellano y Bond (1991), los cuales son apropiados dadas las características de nuestro panel (número

3 Cabe anotar también que a pesar de las expectativas sobre una posible relación positiva entre IED y la explotación de recursos naturales, esto último como proxy de la condición del mercado internacional de commodities, los resultados no las validan. No obstante, es posible que dicha asociación sea más clara si se consideran solo algunos grupos de países, por ejemplo reunidos en el África subsahariana y Latinoamérica, lo cual podría ser objeto de futuros trabajos. 
de países mucho mayor que el de años). Aunque los respectivos resultados no se presentan acá, pues los rezagos de las variables dependientes no fueron significativos $^{4}$ y por las limitaciones de espacio ${ }^{5}$, en general se mantienen los hallazgos previamente comentados, por ejemplo en lo que respecta al crecimiento económico y a la apertura comercial como potenciales atractores de la inversión.

Por otra parte, cabe precisar que el coeficiente que acompaña la estabilidad política presenta un signo contrario al esperado (modelos $1^{*}$ y $2^{*}$ de la Tabla 5), lo que sugiere, de manera contraintuitiva, que la inestabilidad política ceteris paribus se corresponde con un mayor grado de IED. Aunque analizar esto en profundidad queda fuera del alcance de este artículo, es posible que la falta de certidumbre sobre el futuro de las instituciones políticas de un país también suponga una oportunidad de inversión bajo ciertos escenarios, por ejemplo cuando el Gobierno de turno aplica unas políticas que limitan excesivamente el ejercicio del interés privado, como en el caso de los controles a los flujos de capitales.

\section{CONCLUSIONES}

Las reformas tributarias en Colombia, así como en muchos otros países de la región, en los últimos treinta años han hecho énfasis en la necesidad de promo- ver la inversión a través de la reducción de alícuotas del ISRE y la creación de beneficios tributarios que disminuyen la respectiva carga impositiva. Un ejemplo de esto es la reciente Ley de Financiamiento de 2018, que como preceptos asumió que los impuestos que pagaban las empresas en el país eran muy altos, lo que a su vez desincentivaba la inversión y afectaba negativamente el crecimiento económico. En consecuencia, se aprobó una reducción gradual de la tarifa del ISRE para que en 2022 quedara en $30 \%$ y se crearon varias rentas exentas, entre otras la aplicable a la economía naranja.

Sin embargo, en este trabajo se encuentra que la TET relativa al ISRE no es tan alta como se suele indicar por parte del Gobierno y los gremios económicos; por ejemplo, en 2016 se ubicó alrededor del $24 \%$. Pese a lo anterior, la decisión tomada con la Ley de Financiamiento fue reducir el ISRE e incluso ampliar algunos beneficios tributarios (e.g., rentas exentas y descuentos fiscales), con la promesa de que con ello se reactivaría la inversión y en consecuencia la economía, aun cuando la clase media tuviera que compensar la pérdida de recaudo a través de mayores impuestos (vía incrementos del ISRP).

Junto a lo anterior, otro propósito de este trabajo era validar si eventualmente un aumento del ISRE podría dar lugar

$\begin{array}{ll}4 & \text { Lo que además sugiere que la inversión no responde a la dinámica seguida en periodos anteriores. } \\ 5 & \text { Estas estimaciones se podrían suministrar previo requerimiento a los autores. }\end{array}$ 
a una caída de la inversión en general, o de la IED en particular. Al respecto, la evidencia empírica recabada en la sección anterior indica que tanto la IED como la inversión total están relacionadas principalmente con factores relativos a la capacidad de demanda de una economía, el modelo de regulación económica, el grado de apertura comercial y el desarrollo productivo de un país, de modo que los impuestos son relegados en importancia. Los resultados que se muestran en la Tabla 5 sugieren que independientemente de cuál sea la forma como se mida la carga tributaria empresarial, su asociación con la inversión no es estadísticamente significativa.

Cabe aclarar que la literatura teórica y empírica en la materia tampoco es concluyente, pues los resultados suelen depender de los supuestos que se realicen en la modelación, o de la muestra y variables que se utilicen, según el caso. No obstante, desde esta investigación se pueden plantear dos hipótesis que ayudan a explicar la neutralidad de los impuestos empresariales en la inversión. En primer lugar, es de esperar que un inversionista considere con atención las expectativas de rentabilidad que le ofrece un país, lo cual trasciende a la política tributaria, más aun cuando la frecuencia con que se modifica la normativa en casos como el colombiano genera incertidumbre. Asimismo, el esquema de renta global, ampliamente utilizado en el mundo, implica por lo general que las rentas que no se gravan en el país de la fuente deban tributar en el país de domicilio o residencia, lo cual elimina los beneficios que supone el diferencial de las TET entre naciones ${ }^{6}$.

En consecuencia, dos de las principales razones que se arguyen para promover beneficios tributarios en cabeza de las corporaciones (i.e., la alta carga tributaria empresarial y el desincentivo que ello supone para la inversión) quedan sin sustento. Esto invita a repensar los fundamentos bajo los cuales tradicionalmente se reforma un sistema tributario en un país como Colombia, de modo que se empiecen a considerar medidas como la eliminación o limitación de muchos beneficios tributarios que subsisten sin la debida evaluación por parte del Gobierno.

\section{DECLARACIÓN DE CONFLCITO DE INTERES}

Los autores declaran que no existe conflicto de intereses. Este problema se puede resolver parcialmente, si en los convenios para evitar la doble imposición se incluyen
cláusulas que garanticen la aplicación de los beneficios tributarios que ofrecen los países firmantes. 


\section{REFERENCIAS}

[1] Adam, A., Kammas, P. \& Lagou, A. (2013). The Effect of Globalization on Capital Taxation: What Have we Learned After 20 Years of Empirical Studies? Journal of Macroeconomics, 35, 199-209. https://doi.org/10.1016/j. jmacro.2012.09.003

[2] Agostini, C. \& Jalile, I. (2009). Efectos de los impuestos corporativos en la inversión extranjera en América Latina. Latin American Research Review, 44(2), 84-108. https://doi.org/10.1353/lar.0.0127

[3] Alm, J. (1996). What is an Optimal Tax System. National Tax Journal , 49(1), 117-133.

[4] Arellano, M. \& Bond, S. (1991). Some Tests of Specification for Panel Data: Monte Carlo Evidence and an Application to Employment Equations. The Review of Economic Studies, 58 (2), 277-297. https://doi.org/10.2307/2297968

[5] Atkinson, A. \& Stiglitz, J. (2015). Lectures on Public Economics. Princeton University Press.

[6] Banco Mundial. (2018). Global Investment Competitiveness Report 2017/2018: Foreign Investor Perspectives and Policy Implications. World Bank.

[7] Beck, N. \& Katz, J. (1995). What to do (and not to do) with Time-Series Cross-Section Data. American Political Science Review, 89(3), 634-647. https:// doi.org/10.2307/2082979

[8] Bokpin, A., Mensah, L. \& Asemoah, M. (2015). Foreign Direct Investment and Natural Resources in Africa. Journal of Economic Studies, 42(4), 608-621. https://doi.org/10.1108/JES-01-2014-0023

[9] Castañeda-Rodríguez, V. (2016). Las crisis económicas y el escenario político en la introducción de reformas tributarias. La experiencia argentina (1980-2010) y una constatación para América Latina. Cuadernos de Economía, 35(67), 173206. https://doi.org/10.15446/cuad.econ.v35n67.52466

[10] Castañeda-Rodríguez, V. (2019). The 2016 Tax Reform in Colombia: a Patchwork Quilt. Cuadernos de Administración, 35(65), 72-91. https://doi. org/10.25100/cdea.v35i65.7676 
[11] Chanegriha, M., Stewart, C. \& Tsoukis, C. (2017). Identifying the Robust Economic, Geographical and Political Determinants of FDI: An Extreme Bounds Analysis. Empirical Economics, 52(2), 759-776. https://doi.org/10.1007/ s00181-016-1097-1

[12] Doing Business. (2019, April 27). Doing Business. http://www.doingbusiness. org/en/data/exploreeconomies/colombia\#DB_tax

[13] García, A. (2016, 7 mar.). Los altos impuestos son un suicidio para la economía. El Pais, p. 18.

[14] Gómez, H. \& Steiner, R. (2015). La reforma tributaria y su impacto sobre la tasa efectiva de tributación de las firmas en Colombia. Coyuntura Económica: Investigación Económica y Social, 45(1), 13-44.

[15] Hunady, J. \& Orviska, M. (2014). Determinants of Foreign Direct Investment in EU Countries. Do Corporate Taxes Really Matter? Procedia Economics and Finance, 12, 243-250. https://doi.org/10.1016/S2212-5671(14)00341-4

[16] Jiménez, J. \& Podestá, A. (2009). Inversión, incentivos fiscales y gastos tributarios en América Latina. CEPAL.

[17] Ministerio de Hacienda y Crédito Público (2018, 31 de oct.). Exposición de motivos del proyecto de ley "por la cual se expiden normas de financiamiento para el restablecimiento del equilibrio del presupuesto nacional y se dictan otras disposiciones". MHCP.

[18] Rincón-Castro, H. \& Delgado-Rojas, M. E. (2018) ¿Cuánto tributan efectivamente el consumo, el trabajo y el capital en Colombia? Reportes del Emisor, 229, 5. https://doi.org/10.32468/be.1041

[19] Villabona, J. \& Quimbay, C. (2017). Tasas efectivas del impuesto de renta para sectores de la economía colombiana entre el 2000 y el 2015. Innovar, 27(66), 91-108. https://doi.org/10.15446/innovar.v27n66.66805

[20] Villaverde, J. \& Maza, A. (2015). The Determinants of Inward Foreign Direct Investment: Evidence from the European Regions. International Business Review, 24(2), 209-223. https://doi.org/10.1016/j.ibusrev.2014.07.008 


\section{Anexo 1. Inversión y factores asociados: estimaciones preliminares}

\begin{tabular}{ccccccccc}
\hline Variable endógena & \multicolumn{4}{c}{ IED } & \multicolumn{3}{c}{ Inversión_total } \\
\hline Efectos & $\mathrm{EA}$ & $\mathrm{EF}$ & $\mathrm{EA}$ & $\mathrm{EF}$ & $\mathrm{EA}$ & $\mathrm{EF}$ & $\mathrm{EA}$ & $\mathrm{EF}$ \\
\hline Variable / Modelo & 1 & 2 & 3 & 4 & 5 & 6 & 7 & 8 \\
\hline Carga_tributaria_total & 0.030 & 0.013 & & & 0.046 & -0.011 & & \\
\hline Carga_impuestos_renta & & & 0.042 & 0.039 & & & 0.074 & $0.145^{*}$ \\
\hline Estabilidad_política & -0.126 & -1.002 & -0.039 & -0.824 & 1.128 & 1.308 & 1.153 & 1.133 \\
\hline Efectividad_gobierno & 0.760 & 0.728 & 0.565 & 0.764 & -1.298 & -1.258 & -1.440 & -0.945 \\
\hline Control_corrupción & 0.966 & 0.840 & 0.823 & 0.424 & 2.068 & 2.264 & 1.826 & 2.068 \\
\hline Regulación & -0.306 & $0.927^{*}$ & -0.362 & 0.835 & -0.534 & 0.450 & -0.573 & 0.696 \\
\hline LN(PIB_per_cápita) & $-1.371^{*}$ & 4.168 & -1.131 & 4.324 & -2.222 & 9.606 & -1.895 & 10.500 \\
\hline Crecimiento & $0.228^{* * *}$ & $0.161^{* * *}$ & $0.224^{* * *}$ & $0.164^{* * *}$ & $0.352^{* * *}$ & $0.292^{* * *}$ & $0.357^{* * *}$ & $0.297^{* * *}$ \\
\hline Desempleo & -0.069 & -0.098 & -0.053 & -0.089 & $-0.533^{* * *}$ & $-0.488^{* *}$ & $-0.524^{* * *}$ & $-0.496^{* *}$ \\
\hline LN(Población) & $-0.868^{* * *}$ & $10.515^{*}$ & $-0.794^{* * *}$ & $11.821^{* *}$ & 0.192 & $21.208^{* * *}$ & 0.197 & $20.708^{* * *}$ \\
\hline Apertura & $0.036^{* *}$ & 0.038 & $0.038^{* * *}$ & $0.041^{*}$ & $0.059^{* * *}$ & $0.137^{* * *}$ & $0.063^{* * *}$ & $0.132^{* * *}$ \\
\hline Rentas_naturales & 0.009 & 0.034 & -0.004 & -0.003 & 0.036 & $-0.234^{* *}$ & 0.014 & $-0.215^{* *}$ \\
\hline Educación & $0.034^{*}$ & $0.091^{* *}$ & 0.035 & $0.086^{* *}$ & 0.007 & $0.086^{*}$ & 0.007 & $0.095^{*}$ \\
\hline Agricultura & -0.069 & -0.228 & -0.057 & -0.214 & $-0.293^{* *}$ & $-0.337^{* *}$ & $-0.269^{* *}$ & $-0.337^{* *}$ \\
\hline $\mathrm{N}$ & 622 & 622 & 634 & 634 & 556 & 556 & 568 & 568 \\
\hline r2 & & 0.186 & & 0.179 & & 0.414 & 0.425 \\
\hline
\end{tabular}

Fuente: elaboración propia. ${ }^{*}$ Significativo al $10 \%$, **Significativo al $5 \%, * * *$ Significativo al 1\%. 